\title{
Modélisation numérique du transport sédimentaire sur une plage sableuse
}

\author{
B. Camenen ${ }^{1}$, R. Pedreros ${ }^{2}, H$. Dupuis ${ }^{2}, H$. Howa ${ }^{2}$ et P. Larroudé ${ }^{1}$ \\ I Tel: 33476825060 ; fax: 33476825001 \\ LEGI, BP53, 38041 Grenoble cedex, France \\ camenen@hmg.inpg.fr, larroude@hmg.inpg.fr \\ 2 DGO - UMR CNRS 5805 Av. des Facultés, 33405 Talence Cedex \\ Tel: 33556848849 ; fax: 335568408 ; \\ howa@geocean.u-bordeaux.fr,dupuis@geocean.u-bordeaux.fr, \\ pedreros@geocean.u-bordeaux.fr
}

\section{Résumé}

Cette étude a pour but d'obtenir une procédure de calcul relativement simple d'évolution morphologique permettant une représentation correcte des différents phénomènes et processus liés au transport de sables sur les côtes à moyen terme. L'hydrodynamique est décomposé en trois systèmes (houle, courants dus à la houle, courants dus à la marée) permettant le calcul de l'évolution totale des fonds. Cette procédure quasi-permanente est basée sur un modèle 2DH (code de calcul TELEMAC). Les premiers résultats donnent une bonne représentation de l'évolution de la houle et des effets de la marée comparé aux mesures de terrain sur la plage du Trucvert. Il süusiste toutefois quelques problèmies pour l'intégration des contraintes de radiations données par le module de houle dans le module de courant Sont discutés les difficultés dus aux intéractions entre les trois modules et au choix de la formule de transport la plus adaptée pour chacun des calculs.

\begin{abstract}
A model of morphological evolutions in the coastal zone is presented. It is based on $2 \mathrm{DH}$ formulations of water and sediment motion. The hydrodynamic is decomposed into three systems (waves, wave effects and tidal effects) to compute the total evolution of the bottom. First results show a good representation of the wave evolution and the tidal effects compared to the field measurements in Trucvert beach. Problems remain for using the radiation stress to compute the littoral drift and the undertow. The sediment transport model is tested against academic cases which allow us to compare different analytical transport formulae. We discuss the difficulties due to the different cases of interaction between the simplified hydrodynamic systems and the choice of the most relevant formula for each sand transport computation.
\end{abstract}




\section{Introduction}

Depuis ces quinze dernières années, beaucoup de progrès ont été réalisés pour la modélisation numérique des phénomènes côtiers. Il subsiste toutefois quelques difficultés dans les modèles existants pour relier les modules de houle, de courant et de transport de sédiments (grande variabilité en temps et espace, interaction houle-courant, déferlement, effets de marée ...). Notre objectif est de trouver une procédure permettant de résoudre ces difficultés et d'obtenir ainsi des résultats réalistes. Ainsi, partant des travaux de De Vriend (1986), nous essayons d'améliorer la classique procédure quasi permanente avec un modèle $2 \mathrm{DH}$ (TELEMAC). Comme il est très complexe d'intégrer tous les phénomènes côtiers dans un modèle (voire impossible pour un modèle 2DH), il est nécessaire de décomposer ce système complexe en plusieurs cas plus simples que l'on peut modéliser: courants dus à la houle, courants dus à la marée. Les principales difficultés restantes sont alors l'estimation des différents pas de temps hydrodynamiques et morphodynamiques pour chacun de ces modules et les possibles interactions (houle-courant) ainsi qu'une bonne modélisation du débit solide en fonction de ces nombreux phénomènes. Pour ce dernier cas, nous avons programmé et comparé différentes formules de transport sédimentaire (Bijker, 1967 ; Bailard, 1981 et Dibajnia-Watanabe, 1992).

Cette étude s'insère dans le programme PNEC (Programme National d'Environnement Côtier) dont le principal objectif est la compréhension de la formation et de l'évolution des structures sédimentaires (barres, baines...) et de poser des lois physiques appropriées pour représenter les flux sédimentaires réel enregistrés in-situ. Le site étudié est la piage du Trucvert sur la côte atlantique (près du bassin d'Arcachon. Elle a pour principales caractéristiques une ligne de côte rectiligne d'orientation 20 degrés Nord, une morphologie quasi périodique, une marée faible mais non négligeable pour la problématique de baïnes et une houle importante de secteur W-NW dominant.

\section{Etude de terrain de la plage du Trucvert}

La plage du Trucvert se situe sur la côte atlantique de la flèche du CapFerret près du bassin d'Arcachon (voir figure 1). Les principales caractéristiques de

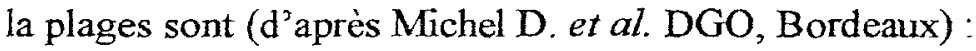

- une plage stable de ligne de côte orientée 20 degrés Nord avec une configuration quasi périodique sur plusieurs kilomètres,

- une marée méso-tidale avec une amplitude de $3.5 \mathrm{~m}$, une légère dissymétrie (5.9 heures pour la marée montante et 6.4 heures pour la marée montante) et créant de faible courants résiduels $(<20 \mathrm{~cm} / \mathrm{s})$,

- une houle de période et amplitude moyennes 8 secondes et 1.5 mètre et de direction principale W-NW.

La caractéristique la plus intéressante de la plage est la rythmicité des barres prélittorales quasi-parallèles à la ligne de côte (légère dissymétrie due à la direction de la houle). Elles créent des bassins plus communément appelés baïnes 
d'une longueur d'environ 400 mètres pour une largeur de 50 mètres et une profondeur de 1.5 mètre. Les dernières campagnes de mesures ont de plus permis d'obtenir une bathymétrie plus précise de la zone subtidale confirmant la présence d'une barre d'avant-côte en croissant (préalablement découverte par observations satellites) d'environ 4 mètres de hauteur, d'une longueur correspondant à 2 ou 3 baines.



Figure 1: Morphologie de la plage du Trucvert (Morphology of Trucvert beach)

\section{Calculs numériques}

Les calculs numériques sur la plage du Trucvert ont été réalisés à l'aide du code calcul TELEMAC (cf. système de modelisation de TELEMAC, 1998). Celui-ci constitue un modèle complet utilisant la méthode éléments finis et permettant la réalisation de différents calculs hydrodynamiques (houle et courants) et sédimentaires. Comme il est encore impossible de modéliser tous les phénomènes se déroulant sur une plage, il peut donc être intéressant de décomposer ce système physique complexe en plusieurs cas plus simples que nous pourrons calculer.

\subsection{Modélisation de l'hydrodynamique}

3.1.1 Calcul de la propagation de la houle avec ARTEMIS 
Le code de calcul ARTEMIS (Agitation and Refraction with TELEMAC on a Mild Slope) résout les équations de Berkoff issues des équations de Navier-Stokes avec les hypothèses supplémentaires d'une faible cambrure de la houle et d'une faible pente du fond. Les principaux résultats sont, pour chaque points du maillage, la hauteur, la phase et l'iucidence de la houle ainsi que le taux de déferlement et les contrainte de radiations.

Pour plusieurs niveau d'eau (suivant la marée), nous avons modélisé l'évolution de la houle. Les graphiques de la figure 2 nous offre un exemple des résultats obtenus avec ARTEMIS. II est ainsi intéressant d'observer l'influence de la barre en croissant créant un cisaillement de la ligne de crête de la houle et l'influence des baines provoquant un second déferlement. Celui-ci semble toutefois être une forme plus numérique puisque la hauteur de la houle est très faible à ce niveau.
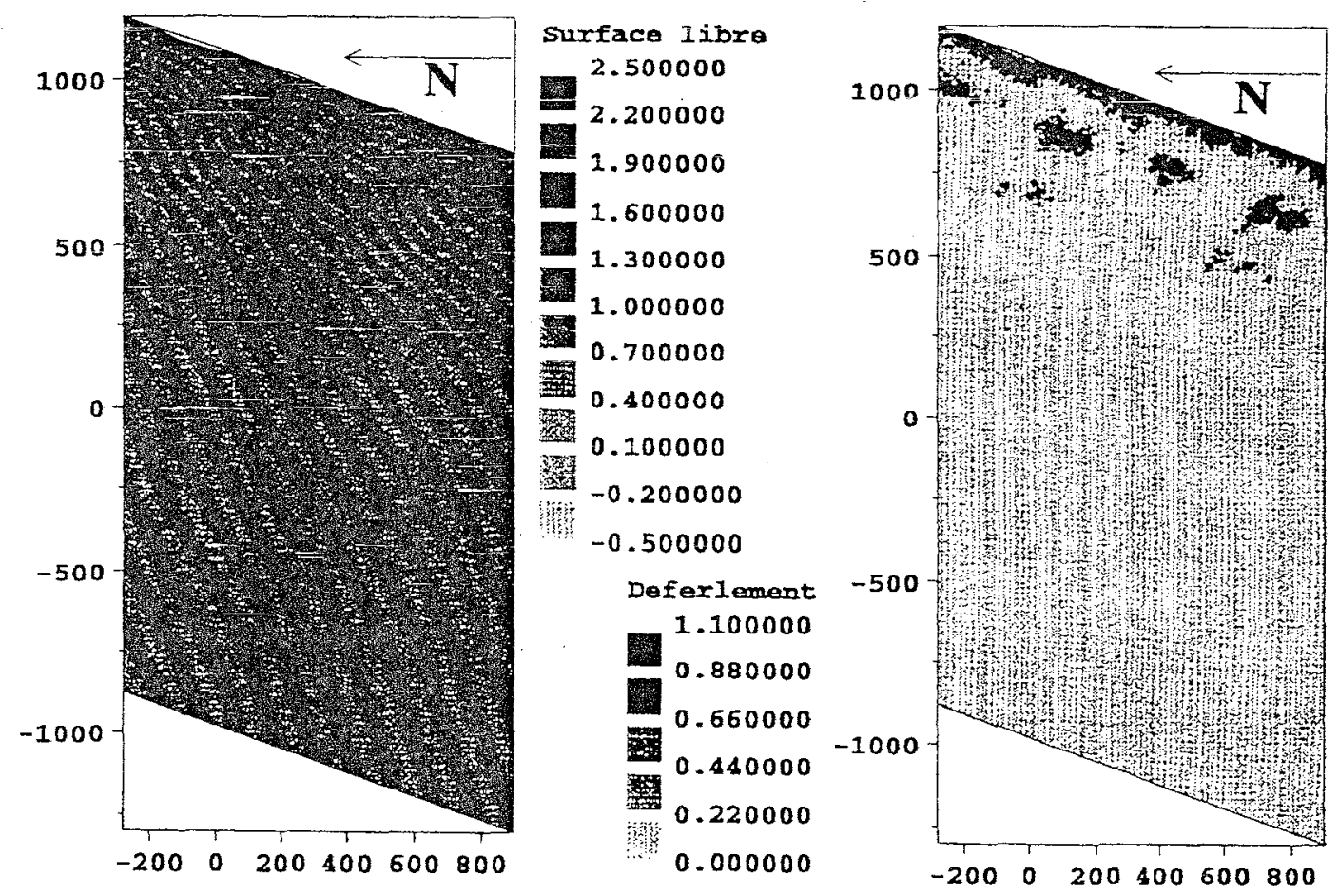

Figure 2 : Surface libre due à la houle et zone de déferlement avec ARTEMIS (Water surface and breaker zone with ARTEMAS)

Ces graphiques nous donnent de plus une bonne vérification de la loi de Snell ( $\mathrm{h} / \sin ^{2} \alpha=$ cst, où $\alpha$ est l'angle d'incidence de la houle), ainsi que de la relation de Miche pour le déferlement $\left(\mathrm{H}_{b}=0,8 . h_{b}\right.$, où $H_{b}$ et $h_{b}$ sont l'amplitude de la houle et la hauteur d'eau au point de déferlement).

Enfin, ARTEMIS donnent des informations fondamentales pour les simulations qui suivent, i.e. la vitesse orbitale et les forces dues aux contraintes de radiation. 


\subsubsection{Calcul de la dérive littorale avec TELEMAC2D}

Le module TELEMAC2D permet la simulation des flux hydrodynamiques en milieu côtier. Il résout les équation de Barré de Saint-Venant (équation de NavierStokes moyennées sur la hauteur). et tient compte entre autre, de possibles bancs découvrants, du frottement de fond et de la turbulence. Les principaux résultats sont, pour chaque points du maillage, la hauteur d'eau et la vitesse moyenne.

Des résultats précédents, nous imposons comme force extérieurs les forces longitudinales dues aux contraintes de radiation $-F_{y}=-\frac{1}{h}\left(\frac{\partial S_{x x}}{\partial x}+\frac{\partial S_{x y}}{\partial y}\right)-$ dans les équations de Saint-Venant et nous prenons comme conditions aux limites le profil de vitesse donné par Longuet-Higgins (1970). Nous obtenons ainsi un modèle correct pour le calcul de la dérive littorale. Nous pensons aussi pouvoir estimer l'untertow" par l'intermédiaire du calcul des contraintes de radiations avec ARTEMIS.
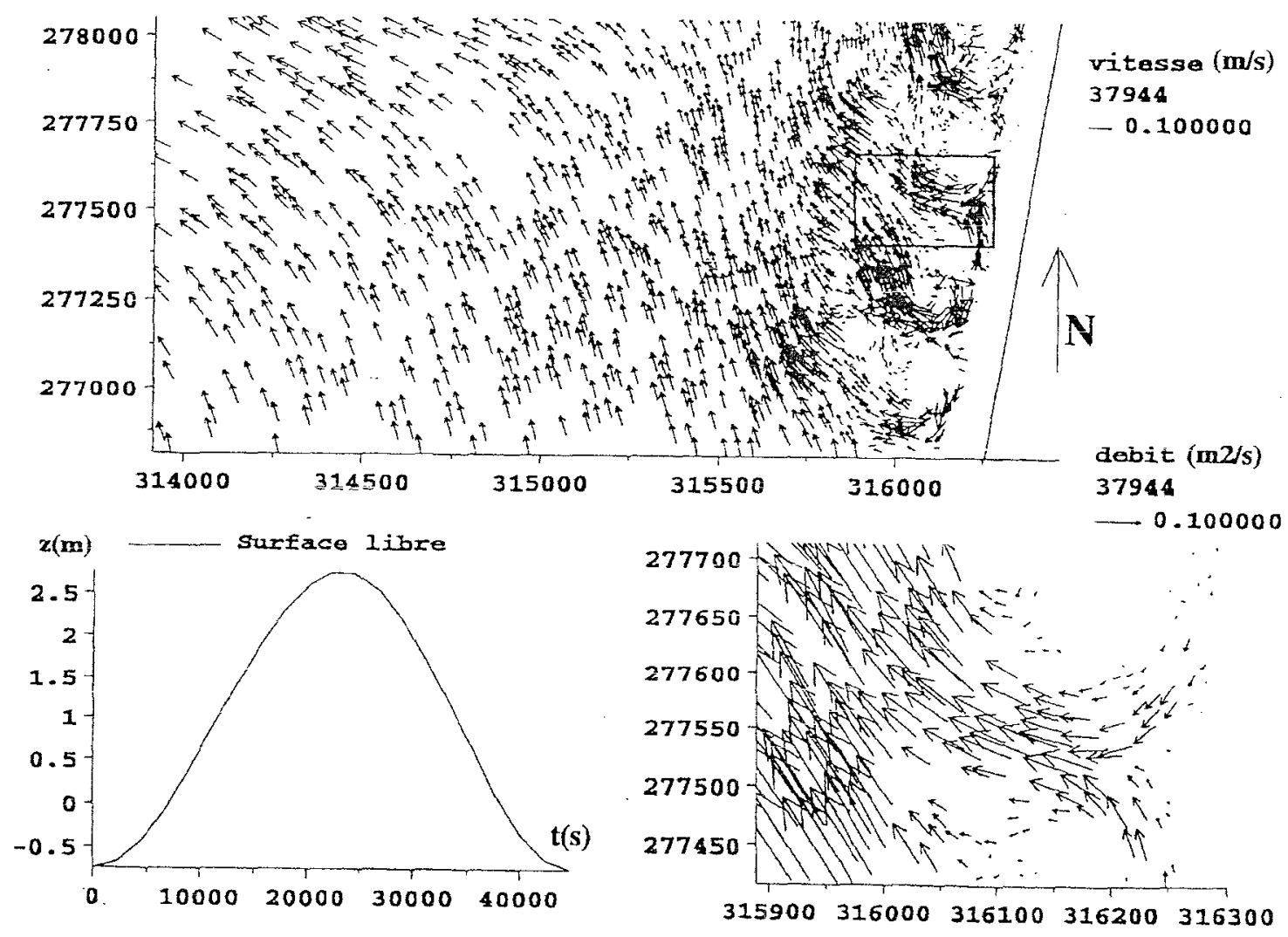

Figure 3: Champ hydrodynamique dû une marée descendante avec TELEMAC2D (Hydrodynamic field, tide going out with TELEMAC2D)

\section{1 .3 Calcul des courants dus à la marée avec TELEMAC2D}

Les courants dus à la marée semble jouer un rôle important dans la formation des baines. Une modélisation de la marée pour un si petit domaine n'est 
pas chose facile. Nous avons donc imposé la hauteur d'eau correspondant à la marée sur la limite au large et une vitesse fonction de la hauteur d'eau et du temps sur les limites "cross-shore" du domaine (courant correspondant du flot et au jusant ainsi qu'au courant résiduel dû à la réfraction de l'onde de marée sur la côte espagnole). La figure 3 montre l'importance de l'influence des baïnes créant une direction privilégiée des courants de marée pendant le montant et le perdant.

\subsection{Modélisation du transport sédimentaire}

Le module SISYPHE résout l'équation de conservation de la masse des fonds en chaque points du maillage:

$$
\frac{\partial . Z_{f}}{\partial . t}+\operatorname{div} \vec{Q}_{s}=0
$$

où Zf est cote du fond et $\vec{Q}_{s}$ est le débit solide volumique calculé à partir des fichiers hydrodynamiques.

Quatre formules semi-empiriques parmi les pius courantes sont déjà intégrées dans le code de calcul (formules de Peter-Meyer, Einstein, Engelund-Hansen et Bijker). Nous avons de plus programmé les formules de Bailard (1981) et DibajniaWatanabe (1992) qui sont plus adaptées au transport sédimentaire sur le littoral (intéraction houle-courant). Enfin, SISYPHE tient compte de l'effet de pente (formulation de Koch et Flokstra, 1981) et offre une méthode de simulation à longterme.

\subsubsection{Formules de transport sédimentaire utilisées}

La première formule utilisée est celle d'Engelund-Hansen (1972) modifiée par Chollet et Cunge (1980) pour prendre en compte les différents régimes de transport solide (régime de dune, "sheet flow"...). Cette formule exprime un transport total uniquement sous un courant permanent. Elle pourra ainsi être utilisée pour le calcul du débit solide sous l'influence de l'"undertow" (pouvant être assimilé à un courant quasi-permanent sur le fond).

$$
Q_{s}=\frac{0.05}{1-n} \sqrt{\frac{(s-1) d^{3}}{g}} K^{2} h^{1 / 3} \tau_{*}^{5 / 2}
$$

où $\mathrm{d}$ est le diamètre du sédiment, $\mathrm{K}$ le coefficient de Strickler de forme, $\mathrm{n}$ la porosité, $\mathrm{s}$ la densité relative du sédiment $(=2.65)$, g l'accélération de la pesanteur, h la hauteur d'eau et $\tau$ * la contrainte de cisaillement adimensionnelle fonction du paramètre de Shields.

L'unique formule tenant compte d'une intéraction entre la houle et le courant déjà intégrée dans le code SISYPHE est la formule de Bijker (1968).

$$
Q_{s}=\frac{5 d}{1-n} \sqrt{\frac{\mu \tau_{c}}{\rho}} \exp \left(-0.27 \frac{\left(\rho_{s}-\rho\right) g d}{\mu \tau_{c w}}\right)\left(1+1.83\left[I_{1} \ln \left(\frac{\sqrt{33 h}}{\delta}\right)+I_{2}\right]\right)
$$

où $\mu$ est un facteur dû à la présence de dunes, $\tau_{c}$ la contrainte de cisaillement due au courant seul, $\tau_{c w}$ la contrainte de cisaillement due à la combinaison de la houle 
et du courant, $\rho_{s}, \rho$ les masses volumiques du sable et de l'eau et $I_{1}, I_{2}$ les intégrales sur la hauteur d'eau des sédiments en suspension (d'après les travaux d'Einstein, 1950).

Nous avons donc intégré au code les formules de Bailard et DibajniaWatanabe permettant une prise en compte de la houle dans le taux de transport mais aussi dans la direction qui n'est alors plus nécessairement identique à celle du courant. La formulation de Bailard tirée de l'approche énergétique de Bagnold (1966) reste l'une des plus utilisée.

$$
\vec{Q}_{s}=\frac{C f}{g(s-1)(1-n)}\left(\frac{\varepsilon_{c}}{\operatorname{tg} \phi}<|\vec{u}|^{2} \vec{u}>+\frac{\varepsilon_{s}}{W_{s}}<|\vec{u}|^{3} \vec{u}>\right)
$$

où $\vec{u}$ est la vitesse combinée houle + courant, $W_{s}, \phi$ la vitesse de chute et l'angle de frottement du sédiment, $\varepsilon_{c}, \varepsilon_{s}$ les efficacités du charriage et de la suspension ( $\left.\varepsilon_{c}=0.2, \varepsilon_{s}=0.025\right), C f$ le coefficient de frottement adimensionnel dû à l'interaction houle-courant et les symboles $\infty$ signifiants une moyenne sur plusieurs période de houle. Le premier terme correspond au transport par chaniage et le second au transport par suspension.

Enfin, la formule de Dibajnia-Watanabe (1992 a une approche très intéressante. Elle décompose en effet le transport en deux demi-cycles dus à la houle. Dans le premier cycle, les sédiments se déplacent dans la direction de la houle et dans le second dans la direction opposée.

$$
\vec{Q}_{s}=0.001 \frac{W_{s} d}{1-n} \frac{\vec{\Gamma}}{\Gamma} \Gamma^{0.55}
$$

où $\vec{\Gamma}$ correspond à la quantité de sédiments transportée sur l'ensemble d'une période de houle en tenant compte de sa direction ainsi que du courant.

\subsubsection{Comparaison de ces formules sur un cas test}

Nous avons comparé ces formules sur un cas test̂́ simple consistant en l'évolution d'une barre sédimentaire le long d'un canal à plafond. La figure 4 nous montre combien ces formules sont sensibles à certains paramètres comme le diamètre du sédiment ou la vitesse orbitale de la houle et peuvent ainsi avoir des comportement très différents. Ainsi, les formules de Bailard et de Bijker sont très sensibles à la taille des grains et estiment une très forte suspension pour les sédiments fins. De même, contrairement aux formules d'Engelund-Hansen et Bijker, les formulations de Bailard et de Dibajnia-Watanabe ne donnent pas un transport nul à partir d'un certain diamètre de particules; ce qui ne semble pas logique si on admet un seuil critique du paramètre de Shields avant mise en mouvement des particules. Enfin, seule la formule de Dibajnia-Watanabe tient compte d'un possible retard de phase du mouvement des sédiments par rapport au mouvement du fluide. Pour de fort vitesse orbitale de la houle ("sheet flow"), le débit solide net aurait ainsi tendance à diminuer, voire même à avoir une direction opposée à l'écoulement moyen dans le cas de sédiment très fins. Ces formulations ont de même une grande influence sur la vitesse de propagation de la barre sédimentaire. Ceci explique donc l'attention toute particulière que nous prêtons au choix de la formule suivant le domaine d'étude. 

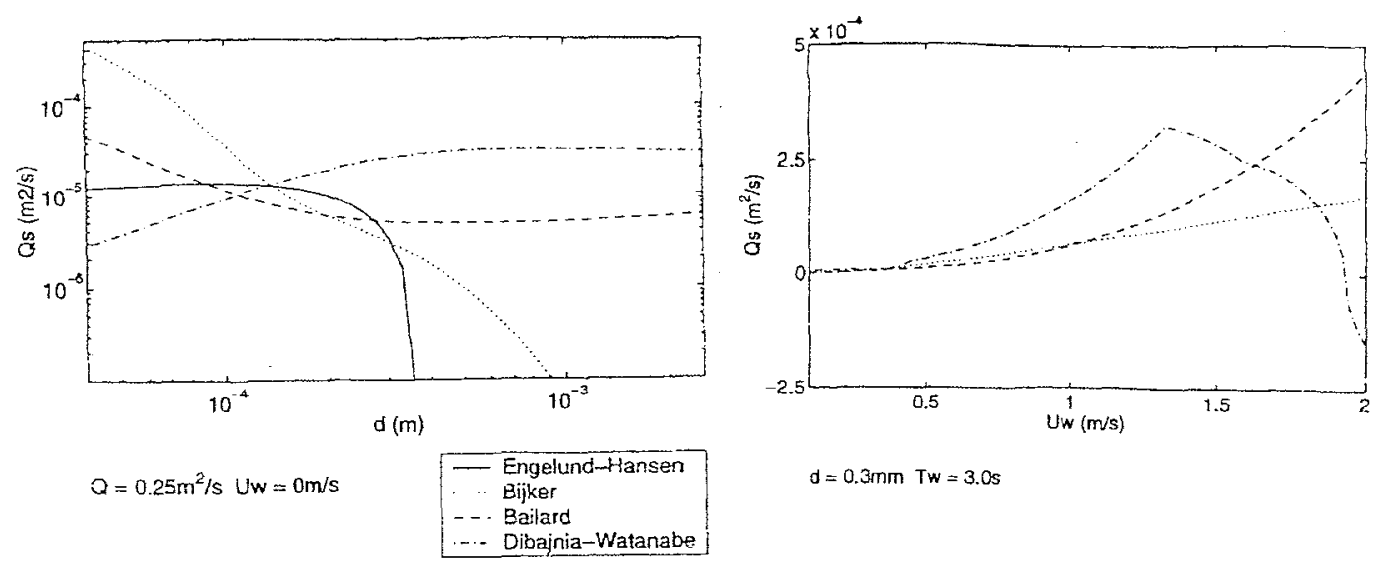

Figure $4:$ Influence du diamètre des grains et de la vitesse orbitale de la houle sur les différentes formules de transport (Grain sand diameter and wave orbital velocity influence on sediment transport formulae)

\subsubsection{Evolution des fonds}

Un des principaux objectifs de notre recherche est d'obtenir une procédure de calcul relativement simple de l'évolution des fonds tout en représentant correctement la réalité. Ainsi, en utilisant le modèle d'instabilité linéaire proposé par A.Falquès et al. (celui-ci nous permet l'obtention d'une bathymétrie initiale comprenant des perturbations), nous cherchons à reconstruire le système de barresbaïnes après qu'une tempête ait aplani la plage. La figure 5 nous donne un premier exemple de calcul. En utilisant les donnés de la plage du Truc-Vert (pente moyenne de la plage, hauteur, période et direction de la houle) et pour une hauteur d'eau donnée, nous avons donc une bathymétrie initiale avec le modèle d'instabilité linéaire. En réduisant l'hydrodynamique au profil de vitesse donné par LonguetHiggins et aux données de houle, nous obtenons de premiers résultats encourageants avec une croissance rapide d'une barre prélittorale asymétrique (utilisation de la formule de Bailard). L'engraissement du bord de plage peu compréhensible et l'instabilité rapide du calcul indiquent toutefois les limites de cette simplification.

Enfin, dans le cas plus complexe où l'on veut tenir compte de la marée, la principale difficulté est alors l'estimation des différents pas de temps entre les modules hydrodynamique et morphodynamique. Pour notre modélisation de la marée, les champs hydrodynamiques obtenus par la simulation ne peuvent être calculés que pour un nombre $\mathrm{n}$ limité de pas de temps de la marée, soit $\mathrm{n}$ niveaux d'eau. Pour chacun de ces niveaux d'eau, il est donc nécessaire de modéliser la propagation de la houle, le courant littoral et le transport sédimentaire résultant de tout ces fichiers hydrodynamiques (pendant un temps $\Delta t$ à déterminer, i.e. après lequel la modification des fonds influence de manière non négligeable l'hydrodynamique). Il est donc extrêmement important de gérer au mieux ce pas de temps pour limiter les coûts de calcul . 


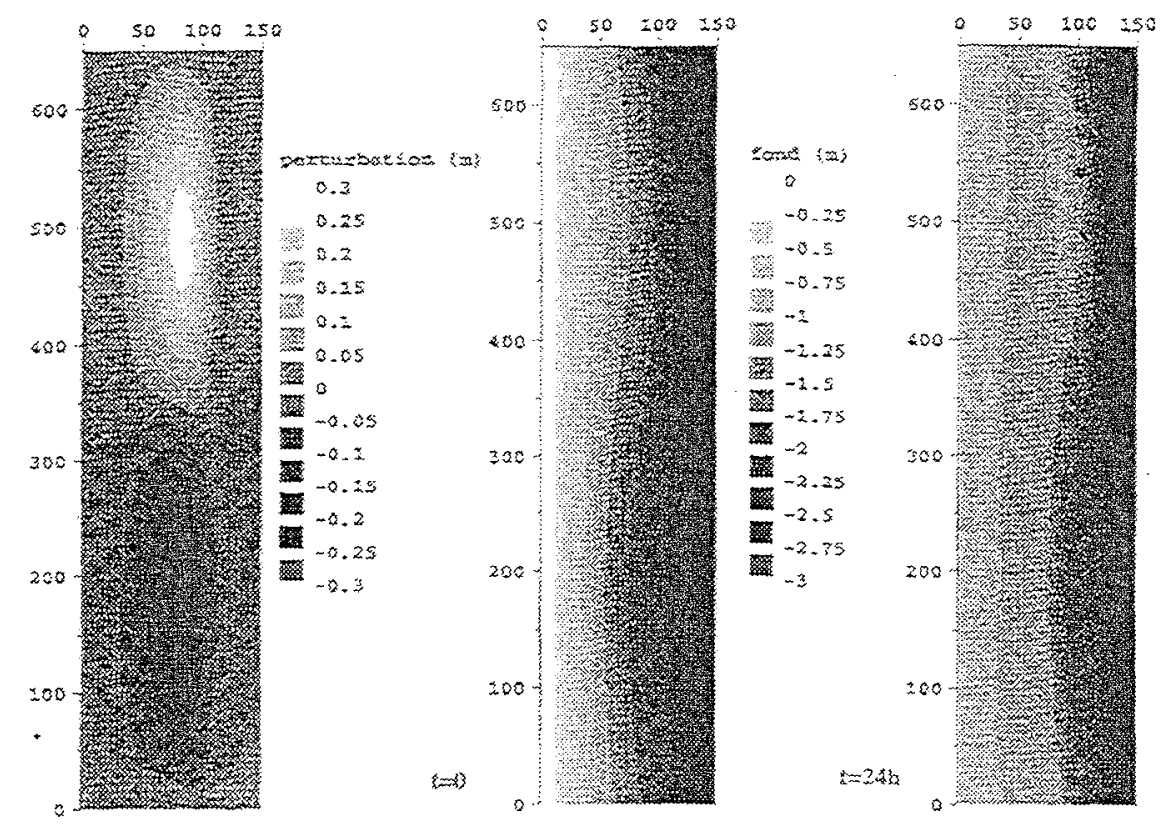

Figure 5 : Exemple de calcul d'évolution des fonds avec SISYPHE en partant des résultats donnés par le modèle de A.Falquès et al (Example of computation of the bottom evolution with SISYPHE software using results taken from the model of A. Falquès et al.)

\section{Conclusion}

Le grand intérêt de cette étude est que nous pourrons ultérieurement comparer nos résultats numériques à l'approche expérimentale sur la plage du Trucvert, en particuliers le transport solide donné par SISYPHE avec celui donné par l'analyse directionnelle de la taille des grains (Pedreros et al., 1996). Ceci devrait nous permettre de moduler nos pas de temps afin d'obtenir les résultats les plus réalistes possibles et surtout de vérifier si le comportement de notre modèle est correct. Les premiers résultats, même s'il ne sont pas encore très réalistes, semblent prometteurs quant à la reconstruction des baïnes après le passage d'une tempête. Enfin, nous avons encore à travailler sur le module de l'"undertow" et sur un possible modèle récurent pour tenir compte de la modification de la houle par les courants existants. Décomposer un système hydrodynamique simplifie beaucoup les calculs numériques et peut aussi permettre une meilleur compréhension des phénomènes en jeu mais a aussi pour conséquence d'omettre certains cas d'intéraction. Une grande partie de notre travail futur sera donc de limiter au mieux cette lacune 


\section{$\underline{\text { Remerciements }}$}

La majeure partie de cette recherche est supportée par le groupe de travail morphologie côtière du programme PNEC financé par le gouvernement français.

\section{Références}

BAILARD J.A. (1981) An energetic total load sediment transport model for a plane sloping beach, J. Geoph. Res., vol.86 C11 pages 10938-10954,

BIKEER E.W. (1968) Littoral drift as function of woves and current, International Conference on Coastal Engineering,

CAMENEN B. and LARROUDE P. (1999) Nearshore transport modelling: application to Trucvert beach, Genova conference,

CAMENEN B. and LARROUDE P. (20000) Numerical comparison of sediment transport formulae, Marine Sandwave Dynamics, Lille

DIBAJNIA M. et WATANABE A.(1992) Sheet flow under nonlinear waves and currents, Coastal Engineering, pages 2015-2029,

ENGELUND F. et HANSEN E. (1967) A monograph on sediment transport in alluvial streams, $3^{\text {rd }}$ edition, Tech. Univ. Of Denmark, Copenhagen, Denmark,

FALQUES A., RIBAS F., LARROUdE P., MONTOTO A. (1999) Nearshore oblique bar modelling : Trucvert beach, Genova conference,

Koch F.G. et FLOKSTRA C. (1981) Bed levels computations for curved alluvial channels, $19^{\text {th }}$ Congress of IAHR, New Delhi, India,

LONGUET-HYGGINS M.S. (1970) Longshore currents generated by obliquely incident sea waves, Journal of geophysical research, vol $75, \mathrm{n}^{\circ} 33$, pages 60778 60801 ,

Michel D., Thomas C., Howa H., Chapalann G., Thais L. et Malengro D. (1999) Processus hydrodynamiques et sédimentaires sur le plateau continental interne aquitain, $7^{\mathrm{e} m}$ Congrès Français de Sédimentologie, Livre des Résumés, Publication ASF, Paris, pages 229-230,

PEDREROS R., HOWA H., MTCHEL D. (1996) Application of grain size trend analysis for the determination of sediment transport pathways in interdidal areas, Marine Geology, vol 135, pages 35-49,

Système de modélisation TELEMAC (1998), EDF-LNH, documentation sur les différent modules,

DE VRIEND H.G. (1987) 2DH Mathematical modelling of morphological evolutions in shallow water, Coastal Engineering, 11, 1-27. 